\title{
Description of the Motion of Objects with Sub- and Superluminal Speeds
}

\author{
Janusz Wolny ${ }^{*}$, Radoslaw Strzalka \\ Faculty of Physics and Applied Computer Science, AGH University of Science and Technology, Krakow, Poland \\ Email address: \\ wolny@fis.agh.edu.pl (J. Wolny) \\ ${ }^{*}$ Corresponding author

\section{To cite this article:} \\ Janusz Wolny, Radoslaw Strzalka. Description of the Motion of Objects with Sub- and Superluminal Speeds. American Journal of Physics and \\ Applications. Vol. 8, No. 2, 2020, pp. 25-28. doi: 10.11648/j.ajpa.20200802.12
}

Received: April 20, 2020; Accepted: May 7, 2020; Published: June 4, 2020

\begin{abstract}
In this paper a direct derivation of the dynamics of objects moving with relativistic speeds is presented, based on two assumptions: $(i)$ energy and mass of an object in motion are equivalent (mass-energy equivalence, known in special relativity and confirmed in experiments), (ii) an object can be considered as a variable-mass object with mass increasing with velocity (in some interpretations referred to as relativistic mass). In the presented approach the postulate on the constancy of the speed of light is not necessary. Also, the four-dimensional Minkowski spacetime is not used and no assumptions on symmetries are made. Therefore, it applies for sub- and superluminal speeds with the speed of light in a vacuum $c$ being the critical speed, which separates the two interesting regions of speeds. The solution for $v<_{c}$ is fully equivalent to the results of special relativity (including the energy-momentum invariant), but the new possible solution for $v>c$ opens an unknown and unintuitive behavior, which should be subjected to experimental investigation. In the range of superluminal speeds, a solution in which the energy of the material particle decreases as its speed increases is obtained. The critical speed in media other than a vacuum should be replaced to a speed environment-dependent, other than $c$.
\end{abstract}

Keywords: Special Relativity; Equation of Dynamics; Sub- and Superluminal Speeds

\section{Introduction}

The motion of objects moving in a vacuum at subluminal (slower-than-light) speeds is conventionally described within the 115-year-old special theory of relativity in Minkowski's four-dimensional space (spacetime). Modern special relativity is the theory based on the tensor formalism applied to the four-dimensional Minkowski spacetime [1]. This description uses the unique geometry of the spacetime with a characteristic metric. An important result in this approach is that for a material particle the speed of light $c$ is a limiting and impassable speed. The question arises whether it is possible to extend the equations of dynamics to a speed range above $c$. The answer within the special relativity is: certainly not. However, this possibility is created by a new approach to relativistic dynamics [2], in which the assumption of the maximum speed of light in a vacuum is abandoned and replaced by another assumption about the equivalence of mass and energy. The new theory requires compliance with commonly known results of the special relativity in the range of subluminal speeds, with the possibility of extending this range.

\section{Relativistic Equation of Dynamics}

The new approach to relativistic dynamics presented in [2] is based on two assumptions: (i) mass and energy equivalence and (ii) the well-known in classical and also relativistic physics equation of dynamics for variable mass systems. Assumption $(i)$ has a very solid experimental basis and is also a fundamental statement for special relativity. Since the discovery of the positron in 1932 by Anderson [3] the emission of energy from the matter-antimatter annihilation was observed and measured. Also together with the birth of nuclear physics, the idea of the mass defect (or mass excess) was introduced by Aston in 1927 [4]. By now, the highest accuracy in mass-energy equivalence was measured for the nuclear binding energy of Si and S isotopes [5].

Combining the commonly used equation of dynamics for systems with variable mass with an additional assumption of 
equivalency of mass and energy, a relativistic equation of dynamics can be deduced [2]. The solutions of this equation for vacuum under the assumption $(i)$ give as a result a critical speed for relativistic objects equal to the constant speed of light in a vacuum, $c$. This new approach to relativistic dynamics is, however, fundamentally different from special relativity and opens the possibility of seeking solutions for equations of motion of objects moving at both subcritical and supercritical velocities in relation to $c$. All calculations are carried out in three-dimensional physical space.

The undeniable (not only theoretically, but above all experimentally) feature of bodies in motion is the proportionality of their mass and energy, which can be written as follows:

$$
E=\alpha m
$$

where $E$ is the total energy of the body with the mass in motion equal to $m$. The proportionality factor, $\alpha$, is a parameter independent of the state of motion of the particle. However, one can admit dependence on external factors, such as the type of medium, or the strength of force fields acting in the medium in which the particle moves. The above equation is better known as the famous Einstein equation: $E=m c^{2}$, in which the parameter $\alpha$ is replaced by the square of the speed of light in vacuum, $c^{2}$. Sometimes, this Einstein equation is limited to objects in rest. Within the presented theory, (1) is treated very generally and applies in any reference system, in particular in the inertial laboratory system (LAB), in which the body moves at the speed $v$.

The second assumption of the presented approach is the application of the equation of dynamics for systems with variable mass (commonly accepted for the whole range of speeds) written in the form of time derivative:

$$
\frac{\mathrm{d} \mathbf{p}}{\mathrm{d} t}=\mathbf{F}
$$

where $\mathbf{p}=m \mathbf{v}$ is the momentum vector, $m$ is the velocity-dependent mass of a particle (relativistic mass), $\mathbf{F}$ external force. Equation (2) is not a general equation of motion of a variable mass system. It is only a special form (see [2]). Here, the dynamics of a variable mass system, where the total mass of a system is constant, is considered.

The presented approach to relativistic dynamics is based on two equations: (1) and (2). After replacing the momentum with the expression $m \mathbf{v}$ in (2), one gets an alternative form of the dynamics equation for a particle moving with acceleration $\mathrm{d} \mathbf{v} / \mathrm{d} t$, under the force $\mathrm{F}$ :

$$
m \frac{\mathrm{d} \mathbf{v}}{\mathrm{d} t}=\mathbf{F}-\frac{\mathrm{d} m}{\mathrm{~d} t} \mathbf{v}
$$

To calculate the rate of mass change the work-energy theorem $(\mathrm{d} E=\mathbf{F} \cdot \mathbf{v} \cdot \mathrm{d} t)$ is used, which gives:

$$
\frac{\mathrm{d} m}{\mathrm{~d} t}=\frac{1}{\alpha} \frac{\mathrm{d} E}{\mathrm{~d} t}=\frac{\mathbf{F} \cdot \mathbf{v}}{\alpha}
$$

By inserting (4) into (3) the equation of relativistic dynamics is obtained:

$$
m \frac{\mathrm{d} \mathbf{v}}{\mathrm{d} t}=\mathbf{F}-\frac{\mathbf{F} \cdot \mathbf{v}}{\alpha} \mathbf{v}
$$

Two components of the force - parallel and perpendicular to the velocity vector, $\mathbf{F}=\left(F_{\|}, F_{\perp}\right)$, with $\mathbf{v}=\left(v_{\|}, v_{\perp}\right)=$ $(v, 0)$ and an acceleration $\mathbf{a}=\left(a_{\|}, a_{\perp}\right)$, are considered. For such reference frame (5) takes the form:

$$
m\left(a_{\|}, a_{\perp}\right)=\left(F_{\|}\left(1-\frac{v^{2}}{\alpha}\right), F_{\perp}\right)
$$

Equation (6) has a stable solution, $a_{\|}=0$, for a certain critical speed, $v_{\mathrm{c}}$, satisfying the relationship: $v_{\mathrm{c}}=\sqrt{\alpha}$. The discussed equation predicts the existence of critical speed for which any force cannot accelerate a particle by changing the length of its velocity vector. Change in energy (and, at the same time, the length of the velocity vector) can be caused by parallel component of the force only. For the vacuum, the critical speed is equal to $c$ satisfying the relationship: $c^{2}=\alpha$, which is called the speed of light in a vacuum, what remains in agreement with special relativity, and is confirmed by a number of experimental results.

The remaining part of this paper concerns the vacuum (assuming $\alpha=c^{2}$ ). According to (5), the dynamics equation after expressing mass by energy, $m=E / c^{2}$, takes the form:

$$
\frac{E}{c^{2}} \frac{\mathrm{d} \mathbf{v}}{\mathrm{d} t}=\mathbf{F}-\frac{\mathbf{F} \cdot \mathbf{v}}{c^{2}} \mathbf{v}
$$

Equation (7) is the equation of motion in the special relativity $[6,7]$. It is a general equation of motion of any physical object with energy $E$ moving under the influence of the force $\mathbf{F}$.

The only assumption used for its derivation is the linear dependence of mass and energy, which, so far, has a very solid experimental basis. If, however, it would turn out that the relationship between energy and mass should also contain additional nonlinear corrections, one has to go back to (1) and include corrections in the relativistic equations of motion. However, the linear relationship between mass and energy is sufficient to reproduce the relativistic equation of dynamics expressed by (7).

\section{Solutions of the Equation of Dynamics}

Now, a closer look at some of the solutions of the relativistic equation of dynamics can be tried. Introducing $\gamma$ as a commonly used relativistic factor defined by the formula:

$$
\gamma \equiv \frac{1}{\sqrt{1-\frac{v^{2}}{c^{2}}}}
$$

one obtains from (7) the following:

$$
\frac{E}{c^{2}} \frac{d v}{d t}=\frac{F_{\|}}{\gamma^{2}} m
$$

Using the work-energy theorem $\left(\mathrm{d} E=F_{\|} v \mathrm{~d} t\right),(9)$ can be also written as:

$$
\frac{v \mathrm{~d} v}{c^{2}\left(1-\left(\frac{v}{c}\right)^{2}\right)}=\frac{\mathrm{d} E}{E}
$$

In (7) and (10) the speed of light in vacuum occurs as a critical speed for which a particle subjected to an external force does not accelerate. Now the solutions of (10) in two separate speed ranges: below $c$ and above $c$ can be 
considered.

\subsection{Velocities Below the Critical Values $(v<c)$}

To solve the differential equation (10) integration over $v$ between limits 0 and $v$ must be performed. The corresponding energy change is from the initial energy $E_{0}$ (at speed 0 , it is the so-called rest energy), to instantaneous energy $E$ (at speed $v$ ). After integration one gets:

$$
-\frac{1}{2} \ln \left|1-\left(\frac{v}{c}\right)^{2}\right|=\ln \left(\frac{E}{E_{0}}\right)
$$

The equation (11) for $v<c$ can be written as:

$$
E(v)=E_{0} \gamma=\frac{E_{0}}{\sqrt{1-\left(\frac{v}{c}\right)^{2}}}
$$

where $E$ is the energy in motion, characteristic for the coordinate system used to describe the phenomenon; $E_{0}$ is rest energy, in the own reference system of the considered object.

Replacing energies by masses $\left(E=m c^{2}\right.$ and $\left.E_{0}=m_{0} c^{2}\right)$, a commonly known relation between the mass in motion $(m)$ and rest mass $\left(m_{0}\right)$, i.e. the relativistic mass ${ }^{1}$ is obtained:

$$
m(v)=m_{0} \gamma=\frac{m_{0}}{\sqrt{1-\left(\frac{v}{c}\right)^{2}}}
$$

By transforming (13) one gets the relativistic energy-momentum invariant:

$$
E^{2}-p^{2} c^{2}=E_{0}^{2}=m_{0}^{2} c^{4}
$$

where the momentum of an object is: $p \equiv m v$.

From (6) and (13) the equation of motion for an object with rest mass $m_{0}$ moving in a vacuum with a speed $v$ under the longitudinal force $F_{\|}$takes the following form:

$$
m_{0} \frac{\mathrm{d} v}{\mathrm{~d} t}=\frac{F_{\|}}{\gamma^{3}}
$$

Equation (15) is a relativistic equation of motion of a particle with rest mass $m_{0}$ moving with velocity $\mathbf{v}$ under the force parallel to the velocity vector.

\subsection{Velocities Above the Critical Speed $(v>c)$ - Reality or Fiction}

Since, within the theory presented all limitations as to the range of permitted speeds are given up, the case of speeds greater than the critical speed $c$ can also be considered. Such the hypothetical possibility was largely discussed in the

\footnotetext{
1 The concept of 'relativistic mass' is questioned by some authors, who sugges that this term introduces lot of misunderstandings (see [8] and series of interesting comments in May 1990 volume of Physics Today under the title Putting to rest mass misconceptions.) Einstein himself was not fond of the expression: $m=E / c^{2}$ (with $m$ being the relativistic mass, and $E$ - total energy) and the term 'relativistic mass'. The term $m_{0} /\left(1-v^{2} / c^{2}\right)^{1 / 2}$ is well supported by the results of the Energy-momentum four-vector in Minkowski space (called also Lorentz-covariant formulation). However, since the early stages of the development of the theory of special relativity the alternative to the four-vector description was developed by introducing the concept of the 'relativistic mass' $[9,10]$. The concept was further developed by modern scientists (e.g. [11]), and is still present in many lectures and textbooks (see, again the argument in Physics Today, May 1990 volume).
}

literature, also quite recently [12-16]. To solve the problem, equation (10) must be reconsidered, a solution of which can now be written in the form:

$$
-\left.\frac{1}{2} \ln \left[\left(\frac{v}{c}\right)^{2}-1\right]\right|_{v_{i}} ^{v}=\left.\ln (E)\right|_{E_{i}} ^{E}
$$

what is:

$$
\frac{E}{E_{i}}=\frac{m}{m_{i}}=\frac{\sqrt{\left(\frac{v_{i}}{c}\right)^{2}-1}}{\sqrt{\left(\frac{v}{c}\right)^{2}-1}}=\frac{\gamma^{\prime}}{\gamma^{\prime} i}
$$

where: $E(m)$ and $E_{i}\left(m_{i}\right)$ is energy (mass) of a particle at actual speed $v$ and initial speed $v_{i}\left(v_{i}>c\right)$, respectively:

$$
\gamma^{\prime} \equiv \frac{1}{\sqrt{\left(\frac{v}{c}\right)^{2}-1}} \text {, and } \gamma_{i}^{\prime} \equiv \frac{1}{\sqrt{\left(\frac{v_{i}}{c}\right)^{2}-1}} .
$$

Equation (17) can also be written in the form:

$$
p^{2} c^{2}-E^{2}=p_{i}^{2} c^{2}-E_{i}^{2}=\text { const }
$$

Equation (19) is analog to the energy-momentum invariant (conservation of the four-vector momentum) for superluminal speeds, $v>c$. Obtained solutions can always be rejected, treating them as non-physical. The final decision, however, should be determined by the results of observations possibly carried out for particles moving at a speed higher than $c$.

Figure 1 presents the energy (and also mass in motion) dependence on speed for the entire velocity area, both subcritical, $v<c$, given by (12) and supercritical $v>c$, given by (17). What is unusual in the behavior of particles for superluminal speeds is the decrease in energy as the speed increases. The particle increases its speed to infinity while reducing its energy.

Below the critical speed the dynamics of the material particle is the same as in special relativity. For superluminal speeds, the particle's behavior becomes completely non-classical: the result of lowering its energy is an increase in the particle's speed to infinity (something like a rapid escape of a particle from the place of the incident). Since there are no relativistic theories for $v>c$, the possible acceptation of the solution presented on the chart requires careful experimental verification.

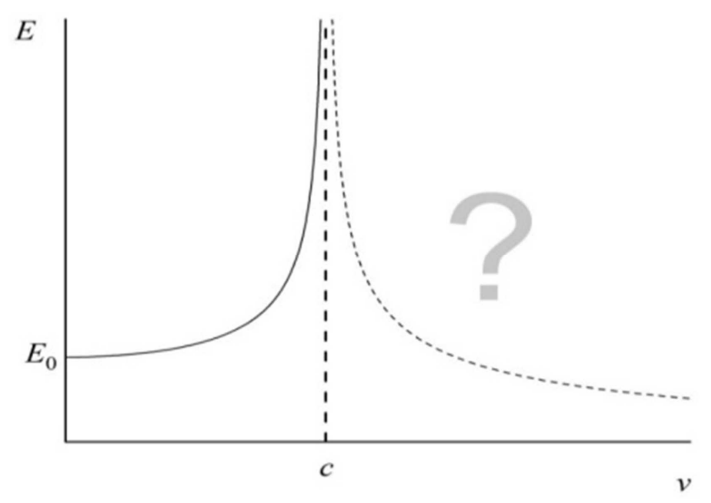

Figure 1. The dependence of energy on the speed of a material object in a full range of speeds (slower-and faster-than-light), obtained on the basis of the assumption of proportionality of mass and energy. The speed of light in a vacuum becomes a critical (asymptotic) speed. 


\section{Summary}

The conclusion from the derivation above is the following. The critical dependence of mass on velocity and the relativistic energy-momentum invariant are the direct results of the dynamical equation for systems with variable mass with the additional assumption of the mass-energy equivalence. One of the key relations of the theory of special relativity, energy-momentum invariant, is a direct conclusion from the presented theory.

The obtained equation (10) leads to independent solutions in two separate regions: sub- and superluminal speeds, which are separated by an asymptote when $v=c$ (for a vacuum). The stationary solution is the critical velocity, which for a vacuum gives the speed of light, $c$. In other media, the critical velocities of moving objects can vary depending on the interaction with the medium. For a speed equal to $c$, both energy and mass behave critically, which causes complete separation of particles with subcritical and supercritical speeds. The transition of particles from one speed region to another is classically impossible. It could occur in the case of blurring of singularity, e.g. as a result of the interaction of the considered particle with the environment, which could affect the modification of the parameter $\alpha$. Another explanation could be a tunneling through the energy barrier or the use of virtual intermediate states. For now, it is too early to indicate whether such a transition between areas of different speeds occurs and which mechanism is possible to implement. From the point of view of observation, one thing seems certain: such transitions are rare, if any, and the observation of particles at superluminal speeds requires exceptional experimental diligence, due to their rapid escape from the place of the incident.

The presented approach to relativistic dynamics is based on two assumptions: the proportionality of mass in motion to the total energy of the body and the equation of motion of objects with variable mass. The equation of motion obtained in this way, in the case of vacuum, is identical to the equation used in special relativity for $v<c$. The speed of light in a vacuum becomes critical speed. At the same time, a new, until now completely inaccessible section of the dynamics of objects moving at supercritical speeds, i.e. above the speed of light, is opened. In the range of superluminal speeds a solution in which the energy of the material particle decreases as its speed increases is obtained. Such particles quickly escape from the place of the incident due to reaching high speeds. Particles at superluminal speeds would be very difficult to detect experimentally. An alternative interpretation is an arbitrary statement that for $v>c$ one gets the solutions that are non-physical and should be rejected. The final decision should be made on the basis of eventual observation of "hyper-relativistic" particles or exclusion of such.

The proposed theory can be used to describe the dynamics of objects in the whole range of velocities: not only for subluminal speed but also for whose velocities which have eventually exceeded critical values for a given media (Cherenkov radiation, tachyons, exotic matter, etc. [17-19]).

\section{References}

[1] Minkowski H. (1907/15). Das Relativitätsprinzip. Ann. Phys. 352, 927-938.

[2] Wolny J., Strzalka R. (2019). Momentum in the dynamics of variable-mass systems: classical and relativistic case. Acta Phys. Pol. A 135, 475-479.

[3] Anderson C. D. (1933). The Positive Electron. Phys. Rev. 43, 491-494.

[4] Aston F. W. (1927). Bakerian Lecture. A New Mass-Spectrograph and the Whole Number Rule. Proc. Roy. Soc. A 115, 487-518.

[5] Rainville S., Thompson J. K., Myers E. G., Brown J. M., Dewey M. S., Kessler Jr. E. G. et al. (2005). A direct test of $\mathrm{E}=\mathrm{mc}^{2}$. Nature 438, 1096-1097.

[6] W. Rindler, Essential Relativity: Special, General, and Cosmological, $2^{\text {nd }}$ ed., OUP Oxford, 2006.

[7] R. Resnick, Introduction to Special Relativity, Wiley, 1968.

[8] Okun L. B. (1968). The Concept of Mass. Physics Today 42, 31-36.

[9] Lewis G. N., Tolman R. C. (1909). The Principle of Relativity, and Non-Newtonian Mechanics. Proc. Am. Acad. Arts \& Sci. 44, 709-726.

[10] Tolman R. (1912). Non-Newtonian Mechanics. The Mass of a Moving Body. Philos. Mag. 23, 375-380.

[11] R. P. Feynman, R. B. Leighton, M. Sands, The Feynman Lectures on Physics, chapters 15-1 and 15-8, online access: http://www.feynmanlectures.caltech.edu/

[12] Feinberg G., Possibility of Faster-Than-Light Particles, Phys. Rev., 1967, 159, 1089-1105.

[13] Heim B. (1977). Vorschlag eines Weges einer einheitlichen Beschreibung der Elementarteilchen [Recommendation of a Way to a Unified Description of Elementary Particles]. Zeitschrift für Naturforschung 32a, 233-243.

[14] Gonzalez-Diaz P. F. (2000). Warp drive space-time. Phys. Rev. D 62, 044005.

[15] Wang L. J., Kuzmich A., Dogariu A. (2000). Gain-assisted superluminal light propagation. Nature 406, 277-279.

[16] Zhang S., Chen J. F., Liu C., Loy M. M. T., Wong G. K. L., Du S. (2011). Optical Precursor of a Single Photon. Phys. Rev. Lett. $106,243602$.

[17] Chodos A., Kostelecký V. A., Potting R., Gates E. (1992). Null experiments for neutrino masses, Mod. Phys. Lett. A 7, 467-476.

[18] Chang T. (2002). Parity Violation and Neutrino Mass. Nucl. Sci. Tech. 13, 129-133.

[19] Wang Z. Y. (2016). Modern Theory for Electromagnetic Metamaterials. Plasmonics 11, 503-508. 